\title{
Computing radiation from Kerr black holes: Generalization of the Sasaki-Nakamura equation
}

\author{
Scott A. Hughes \\ Theoretical Astrophysics, California Institute of Technology, Pasadena, CA 91125
}

\begin{abstract}
As shown by Teukolsky, the master equation governing the propagation of weak radiation in a black hole spacetime can be separated into four ordinary differential equations, one for each spacetime coordinate. ("Weak" means the radiation's amplitude is small enough that its own gravitation may be neglected.) Unfortunately, it is difficult to accurately compute solutions to the separated radial equation (the Teukolsky equation), particularly in a numerical implementation. The fundamental reason for this is that the Teukolsky equation's potentials are long ranged. For non-spinning black holes, one can get around this difficulty by applying transformations which relate the Teukolsky solution to solutions of the Regge-Wheeler equation, which has a short-ranged potential. A particularly attractive generalization of this approach to spinning black holes for gravitational radiation (spin weight $s=-2$ ) was given by Sasaki and Nakamura. In this paper, I generalize Sasaki and Nakamura's results to encompass radiation fields of arbitrary integer spin weight, and give results directly applicable to scalar $(s=0)$ and electromagnetic $(s=-1)$ radiation. These results may be of interest for studies of astrophysical radiation processes near black holes, and of programs to compute radiation reaction forces in curved spacetime.
\end{abstract}

PACS number: $04.25 . \mathrm{Nx}$

\section{INTRODUCTION}

In 1973, Teukolsky [1] derived a single partial differential equation describing the evolution of perturbations to rotating (Kerr) black holes. This master equation gives the linearized evolution of fields that arise from a perturbing source of stress energy - the charge and current densities associated with the perturbation — to the (vacuum) black hole background. The solutions of the homogeneous version of this equation describe the propagation of radiation in black hole spacetimes. Thus, a common use of this formalism is to study the radiation emitted by matter in the environment of a black hole. In some cases, one can use such an analysis to study back reaction, determining how the perturbing source evolves as radiation carries away energy and angular momentum. A beautiful feature of the master equation is that it describes radiation fields of arbitrary spin weight $s$. It has been used extensively to study scalar $(s=0)$, electromagnetic $(s= \pm 1)$, and gravitational $(s= \pm 2)$ radiation in Kerr spacetimes.

The master equation is often[ solved by introducing a multipolar decomposition of the radiation field. The solution separates into functions of the Boyer-Lindquist coordinates:

$$
{ }_{s} \Psi=\sum_{l, m, \omega} R_{l m \omega}(r)_{s} S_{l m}^{a \omega}(\theta) e^{i m \phi} e^{-i \omega t} ;
$$

each function is governed by an ordinary differential equation. (The precise meaning of ${ }_{s} \Psi$ is described in Sec. [1].) The $t$ and $\phi$ dependences are trivial. The $\theta$ dependence is more involved, but can be evaluated in a straightforward matter. The functions ${ }_{s} S_{l m}^{a \omega}$ are spin-weighted spheroidal harmonics, which are generalizations of spin-weighted spherical harmonics to a spheroidal geometry. The spin-weighted spherical harmonics in turn are generalizations of spherical harmonics that encode the rotation properties of spin $s$ fields; see Refs. [3, 1 (1) for further discussion. A detailed algorithm for computing ${ }_{s} S_{l m}^{a \omega}(\theta)$ is given in Ref. [5]. For the purposes of this paper, the $\theta$ dependence is considered known.

The radial dependence, $R_{\operatorname{lm} \omega}(r) \equiv R(r)$, on the other hand, can be rather difficult to calculate in practice, particularly in a numerical computation. The fundamental reason for this difficulty is the nature of the equation that governs $R(r)$ : this equation (the Teukolsky equation) has a long-ranged potential. In source-free form, it can be written

$$
\frac{d^{2} R}{d r^{* 2}}+F_{T}(r) \frac{d R}{d r^{*}}+\left[\omega^{2}-U_{T}(r)\right] R=0,
$$

\footnotetext{
${ }^{1}$ Separation is not always used. There is also a body of work that uses the master equation to evolve initial data. This approach has been extensively used to study the endpoint of binary black hole collisions; see Ref. [2] and references therein.
} 
where $\omega$ is the frequency of the radiation mode and

$$
r^{*}=r+\frac{2 M r_{+}}{r_{+}-r_{-}} \ln \frac{r-r_{+}}{2 M}-\frac{2 M r_{-}}{r_{+}-r_{-}} \ln \frac{r-r_{-}}{2 M}
$$

is the Kerr "tortoise coordinate". The potentials $U_{T}(r)$ and $F_{T}(r)$ are rather complicated; they encode the most interesting features of wave propagation in black hole spacetimes, such as scatter from spacetime curvature ${ }^{2}$ and superradiant scattering (radiation whose scattered amplitude exceeds the ingoing amplitude due to extraction of energy from the black hole's spin). For large $r$,

$$
\begin{aligned}
& F_{T}(r)=\frac{2(1+s)}{r}-\frac{2 M(2+s)}{r^{2}}+O\left(1 / r^{3}\right), \\
& U_{T}(r)=-\frac{4 i s \omega}{r}+\frac{\lambda+2 a m \omega+8 I M s \omega}{r^{2}}+O\left(1 / r^{3}\right) .
\end{aligned}
$$

[The quantity $\lambda$ is related to the eigenvalues of the $\theta$ dependence; see Ref. [5] for details.] For large $r, F_{T}(r)$ and $U_{T}(r)$ fall off only as $1 / r$ - they are long-ranged, like the Coulomb potential. The solution of Eq. (1.2) for large $r$ is [7]

$$
R=C_{1} \frac{e^{-i \omega r^{*}}}{r}+C_{2} \frac{e^{i \omega r^{*}}}{r^{2 s+1}} .
$$

The complex constants $C_{1}$ and $C_{2}$ are determined by boundary conditions. This asymptotic solution illustrates the difficulty in solving the Teukolsky equation: the coefficient of $e^{i \omega r^{*}}$ differs from the coefficient of $e^{-i \omega r^{*}}$ by $r^{-2 s}$. For negative $s$, this becomes extremely large - large enough that the ingoing $e^{-i \omega r^{*}}$ piece will eventually be entirely lost in any numerical computation due to round-off error. Hence, for negative $s$, it is nearly impossible to set proper boundary conditions on the solution's phase at large $r$. Similarly, for positive $s$ it is difficult to set boundary conditions near the event horizon 1 . For $r$ very close to $r_{+}=M+\sqrt{M^{2}-a^{2}}$ (the location of the event horizon in Boyer-Lindquist coordinates), the solution is [7]

$$
R=C_{3} \Delta^{-s} e^{-i p r^{*}}+C_{4} e^{i p r^{*}} .
$$

I have introduced $p=\omega-m \omega_{+}$, where $\omega_{+}=a / 2 M r_{+}$is the angular velocity at which observers at the horizon are seen to rotate. Because the Boyer-Lindquist coordinates $t$ and $\phi$ become twisted and entangled near the horizon, $p$ describes the frequency of wave modes in that region. The factor $\Delta=r^{2}-2 M r+a^{2}$ goes to zero at the event horizon. Hence, for positive $s$, the ingoing solution swamps the outgoing solution as one approaches the horizon. Whether one chooses positive or negative $s$, there exists a domain in which one cannot accurately compute numerical solutions by directly integrating the homogeneous Teukolsky equation.

Various approaches have been discussed to circumvent this difficulty. One of the first was introduced by Teukolsky and Press [7, 8]. Their approach used the fact that, for a given $|s|$, the solutions to Eq. (1.2) for $s=+|s|$ and $s=-|s|$ are physically equivalent: there exist rules to take the positive $s$ solution to the negative $s$ solution, and vice versa. Thus, one can initially choose $s$ so that the solution is well-behaved in the initial $r$ domain, and then "switch horses" and integrate with the other sign of $s$ as the integration approaches the other asymptotic domain.

A somewhat more elegant way to compute $R$ was developed by Chandrasekhar [9]. As already noted, the poor behavior of the solutions (1.5) and (1.6) is due to the long-ranged nature of the Teukolsky equation's potentials. Rather than try to work with an equation that is simply not well-behaved, one should find transformations which relate the Teukolsky solution $R$ to the solution $X$ of some equation whose potentials are short-ranged. For example, when the black hole spin is zero, black hole perturbations can be described using the generalized Regge-Wheeler equation [10:

$$
\left[\frac{d^{2}}{d r^{* 2}}+\omega^{2}-V_{R W}(r, s)\right] X=0
$$

\footnotetext{
${ }^{2}$ As shown by Leonard and Poisson [6], the phenomenon of tails (delayed propagation due to scatter from spacetime curvature) is to leading order independent of $s$, and is encoded in the logarithmic behavior of $r^{*}$, not the potentials.

${ }^{3}$ One can expand the potentials near the horizon and see that they die away slowly as $r^{*} \rightarrow-\infty$ (which corresponds to $\left.r \rightarrow r_{+}\right)$. The actual form is somewhat messy, and is not given here explicitly.
} 
where

$$
V_{R W}(r, s)=f\left[\frac{l(l+1)}{r^{2}}-\frac{2\left(s^{2}-1\right) M}{r^{3}}\right] .
$$

(Here, $f=1-2 M / r$.) This potential dies away faster than $1 / r$, and so is short ranged. Chandrasekhar showed (for specific choices of $s$ ) that solutions to Eq. (1.7) and solutions to Eq. (1.2) (with $a=0$ ) are related by simple rules. (Below I generalize these rules to any value of s.) This is extremely useful for numerical work: one can integrate Eq. (1.7) to accurately compute $X$ and then transform to $R$. Note that in Ref. [8] Press and Teukolsky introduced a transformation that, in essence, transformed to a function governed by an equation with a better behaved potential. They did not, however, discuss the nature of the transformation in terms of the rangedness of the potentials; Chandrasekhar appears to have been the first to systematically approach this problem with the viewpoint that the long-ranged potential was the key issue. Note also that Chandrasekhar's notation [9] is rather different from that used here; I use a notation similar to that used in [11]. The transformation given in Refs. [9] and [11] is for $s=-2$; a rule for $s=-1$ is given in $[6]$.

For spinning black holes, perhaps the most elegant generalization of Chandrasekhar's approach was given by Sasaki and Nakamura [12]. They derive a transformation rule which relates $R$ for any physical spin $a$ to the solution $X$ of an equation whose potentials are short ranged. The transformation and short-ranged potentials are designed such that if $a=0$, the potentials reduce to the Regge-Wheeler potentials. This approach is very natural in the sense that its solutions are monotonic with respect to spin, ranging from the Schwarzschild value $a=0$ to the extreme Kerr limit $a=M$. Chandrasekhar and Detweiler also investigated several transformations relating $R$ to a short-ranged solution $X$ [13 16]. In some (but not all) cases, these transformations are governed by equations which reduce to the Regge-Wheeler equation in the $a=0$ limit; however, the equations themselves are often not as "nice" to work with. For example, the equation for $X$ is sometimes given in terms of a frequency dependent variable $\hat{r}^{*}(\omega)$ (see Ref. [15]) which is different from the "usual" tortoise coordinate $r^{*}$ [cf. Eq. (1.3)]. This coordinate can be doubly valued and mask features such as superradiant scattering. Also, the potentials of each of their equations are pathological for some set of frequencies [17. By using a set of multiple perturbation equations and transformation laws, one can always find a non-pathological tool for any given frequency. But, there is no single rule that works for all frequencies. (These difficulties do not mean that Chandrasekhar and Detweiler's approaches are not useful. Campanelli and Lousto [18] used rules very similar to Chandrasekhar and Detweiler's in order to show that solutions to the Teukolsky equation are well-behaved even for sources that extend to infinity.)

Sasaki and Nakamura's work is restricted to the choice $s=-2$. This is an appropriate choice for studies of gravitational perturbations, and so the Sasaki-Nakamura equation has been extensively used in studies of gravitational-wave generation and gravitational radiation reaction [5, 12, 19 22. Other values of $s$ are interesting as well. For example, $s= \pm 1$ corresponds to electromagnetic radiation. The propagation and scatter of electromagnetic waves in black hole spacetimes is of great astrophysical interest. Also, much work is currently being directed toward understanding how one calculates self forces and radiation reaction forces in curved spacetimes 23 30]. Implementations of the general formalism (Refs. 23,24]) to date have been restricted to scalar $(s=0)$ or electromagnetic fields. They have also been restricted to spherically symmetric spacetimes. Tools for effective calculation of radiation fields in Kerr spacetimes will make it possible to extend these calculations to more realistic spinning black holes.

In this paper, I generalize the Sasaki-Nakamura equation to arbitrary integer spin weight $s$. The generalized Sasaki-Nakamura (GSN) potentials are given for any $s$, but in terms of two unknown functions, $\alpha(r)$ and $\beta(r)$. These functions are fixed by requiring that the transformation which relates the Teukolsky solution to the GSN solution reduces, in the Schwarzschild limit, to the transformation between the Teukolsky solution and the Regge-Wheeler solution. They also must be chosen so that the potentials they generate are of short range.

There is a great deal of freedom in how one chooses $\alpha$ and $\beta$ : for each value of $s$, there are an infinite number of functions which lead to a transformation with the correct limiting value and that produces short-ranged potentials. It is thus most practical to develop $\alpha$ and $\beta$ on a case by case basis, rather than trying to develop generic formulas. Given the interest in scalar and electromagnetic radiation, I provide examples of $\alpha$ and $\beta$ for $s=0$ and $s=-1$.

Throughout this paper, a prime denotes $d / d r$, where $r$ is either the Boyer-Lindquist or the Schwarzschild coordinate (which coordinate should be clear from context). An overbar denotes complex conjugation. The function $\Delta=$ $r^{2}-2 M r+a^{2}$, and $f=1-2 M / r$. Thus, for Schwarzschild holes, $\Delta=r^{2} f$. The function $R$ will always denote the solution to the homogeneous Teukolsky equation; $X$ will always refer to the solution of the equation with short-ranged potential. Section II reviews important aspects of the Teukolsky equation and its solutions, particularly how one can compute the solution given an appropriate source term and solutions to the homogeneous equation, and how those solutions are related to physical radiation fields. In Sec. III, I review the transformation rules for Schwarzschild black holes. These rules, which are particularly simple, will be used as guidelines for constructing transformations appropriate to radiation in Kerr spacetimes. Finally, in Sec. IV I construct the short-ranged equation for Kerr black 
holes and provide a recipe for specifying the transformation rule for a radiation field of arbitrary spin weight. I apply this recipe in Secs. $\mathrm{V}$ and $\mathrm{VI}$ to scalar and electromagnetic radiation fields, respectively. The resultant transformation rules and equations should form a useful basis for further studies of radiation in Kerr spacetimes. Some concluding discussion is given in Sec. VII.

\section{SOME PROPERTIES OF THE TEUKOLSKY EQUATION AND ITS SOLUTIONS}

As background for the calculations in this paper, I review in this section the most important properties of the Teukolsky equation and its solutions.

As discussed in the Introduction, Teukolsky [1] showed that one can separate the wave equation for a field ${ }_{s} \Psi$ of spin weight $s$ radiation propagating on a Kerr black-hole background using the multipolar decomposition given in Eq. (1.1). The $\phi$ and $t$ dependence is trivial, and the $\theta$ dependence is straightforwardly dealt with. The $r$ dependence, on the other hand, can cause problems.

The radial function $R(r)$ is a solution to the Teukolsky equation, Eq. (1.2). Here I write the Teukolsky equation with its source term and in terms of derivatives with respect to $r$ rather than $r^{*}$ :

$$
\Delta^{-s}\left(\Delta^{s+1} R^{\prime}\right)^{\prime}-V_{T}(r) R=-\mathcal{T}(r) .
$$

This is the way the Teukolsky equation usually appears in the literature. The potential $V_{T}(r)$ is

$$
V_{T}(r)=\lambda-4 i s \omega r-\frac{K(r)^{2}-2 i s(r-M) K(r)}{\Delta} ;
$$

the quantity $\lambda=\mathcal{E}_{l m}-2 a m \omega+a^{2} \omega^{2}-s(s+1)$, where $\mathcal{E}_{l m}$ is the eigenvalue of the spheroidal harmonic [see [5]; in the Schwarzschild limit, $\left.\mathcal{E}_{l m}=l(l+1)\right]$. The function $K(r)=\left(r^{2}+a^{2}\right) \omega-m a$.

The source term $\mathcal{T}(r)$ depends upon the spin weight of the radiation. It is constructed by projecting the radiation source onto legs of the Newman-Penrose null tetrad, $\mathbf{l}, \mathbf{n}, \mathbf{m}$, and $\overline{\mathbf{m}}$. A useful representation of the tetrad in Boyer-Lindquist coordinates is 16 .

$$
\begin{aligned}
l_{\alpha} & =\left[1,-\frac{\Sigma}{\Delta}, 0,-a \sin ^{2} \theta\right], \\
n_{\alpha} & =\frac{1}{2}\left[\frac{\Delta}{\Sigma}, 1,0,-\frac{a \Delta \sin ^{2} \theta}{\Sigma}\right], \\
m_{\alpha} & =\frac{1}{\sqrt{2}(r+i a \cos \theta)}\left[i a \sin \theta, 0,-\Sigma,-i\left(r^{2}+a^{2}\right) \sin \theta\right] .
\end{aligned}
$$

The tetrad legs $\mathbf{l}$ and $\mathbf{n}$ represent ingoing and outgoing null vectors, respectively. Quantities constructed by projecting onto $\mathbf{l}$ correspond to ingoing radiation and their sources; they map to positive $s$. Likewise, projection onto $\mathbf{n}$ corresponds to outgoing radiation and their sources $\$$, and map to negative $s$. See Ref. [1] for details.

Because Eq. (2.1) is in self-adjoint form, one can construct its solution by the method of Green's functions [31]. This means that one needs to know only the solutions to the homogeneous equation,

$$
\Delta^{-s}\left(\Delta^{s+1} R^{\prime}\right)^{\prime}-V_{T}(r) R=0,
$$

in addition to the source. One does this by adapting the generic solution, given in Eqs. (1.5) and (1.6), to the appropriate boundary conditions: no radiation may come in from infinity and none may come out from the event horizon. In other words, there exist two solutions, $R^{H}(r)$ and $R^{\infty}(r)$, whose asymptotic forms are

$$
\begin{aligned}
R^{H}(r) & =B^{\text {hole }} \Delta^{-s} e^{-i p r^{*}}, \quad r \rightarrow r_{+} \\
& =B^{\text {out }} \frac{e^{i \omega r^{*}}}{r^{2 s+1}}+B^{\text {in }} \frac{e^{-i \omega r^{*}}}{r}, \quad r \rightarrow \infty, \\
R^{\infty}(r) & =D^{\text {out }} e^{i p r^{*}}+D^{\text {in }} \Delta^{-s} e^{-i p r^{*}}, \quad r \rightarrow r_{+} \\
& =D^{\infty} \frac{e^{i \omega r^{*}}}{r^{2 s+1}}, \quad r \rightarrow \infty .
\end{aligned}
$$

\footnotetext{
${ }^{4}$ Because one can transform between positive and negative $s$ solutions, one can actually develop both ingoing and and outgoing radiation with a single source term.
} 
The solution to the inhomogeneous equation (2.1) which one constructs from Eqs. (2.5), (2.6), and the source $\mathcal{T}(r)$ is conveniently written

$$
R(r)=Z^{H}(r) R^{\infty}(r)+Z^{\infty}(r) R^{H}(r)
$$

where

$$
\begin{aligned}
& Z^{H}(r)=\frac{1}{2 i \omega B^{\text {in }} D^{\infty}} \int_{r_{+}}^{r} d r^{\prime} \Delta\left(r^{\prime}\right)^{s} R^{H}\left(r^{\prime}\right) \mathcal{T}\left(r^{\prime}\right), \\
& Z^{\infty}(r)=\frac{1}{2 i \omega B^{\text {in }} D^{\infty}} \int_{r}^{\infty} d r^{\prime} \Delta\left(r^{\prime}\right)^{s} R^{\infty}\left(r^{\prime}\right) \mathcal{T}\left(r^{\prime}\right)
\end{aligned}
$$

Using Eq. (2.7) one can construct ${ }_{s} \Psi$. This quantity is related to a radiation field of spin weight $s$; the details of that relation depend upon the value of $s$. Typically, ${ }_{s} \Psi$ is constructed by projecting a tensor describing the radiation onto legs of the Newman-Penrose null tetrad. For example, ${ }_{0} \Psi=\Phi$, a massless scalar field. No projections are needed in this case. For $s= \pm 1$, we have

$$
\begin{aligned}
{ }_{1} \Psi & =\phi_{0}=F_{\mu \nu} l^{\mu} m^{\nu}, \\
{ }_{-1} \Psi & =(r-i a \cos \theta)^{2} \phi_{2}=(r-i a \cos \theta)^{2} F_{\mu \nu} n^{\mu} \bar{m}^{\nu},
\end{aligned}
$$

where $F_{\mu \nu}$ is the electromagnetic field tensor. [There is a third projection, $\phi_{1}=(1 / 2) F_{\mu \nu}\left(l^{\mu} n^{\nu}+\bar{m}^{\mu} m^{\nu}\right)$. It does not describe the radiative degrees of freedom of the electromagnetic field, and so is of less interest here.] For $s= \pm 2$, the radiative quantities are

$$
\begin{aligned}
{ }_{2} \Psi & =\psi_{0}=-C_{\alpha \beta \gamma \delta} l^{\alpha} m^{\beta} l^{\gamma} m^{\delta}, \\
{ }_{-2} \Psi & =(r-i a \cos \theta)^{4} \psi_{4}=-(r-i a \cos \theta)^{4} C_{\alpha \beta \gamma \delta} n^{\alpha} \bar{m}^{\beta} n^{\gamma} \bar{m}^{\delta} .
\end{aligned}
$$

The tensor $C_{\alpha \beta \gamma \delta}$ is the Weyl component of the spacetime's curvature. The quantities $\psi_{i}$, with $i$ an integer from 0 to 4, are the Newman-Penrose projections of the Weyl curvature (see Ref. [16]). For unperturbed black hole spacetimes, all components except $\psi_{2}=-C_{\alpha \beta \gamma \delta} l^{\alpha} m^{\beta} \bar{m}^{\gamma} n^{\delta}=M /(r-i a \cos \theta)^{3}$ can be set to zero with an appropriate choice of gauge. This is the non-radiative "background" component of the curvature; the perturbations $\psi_{0}$ and $\psi_{4}$ represent radiation on the background.

The solution for the (linear) evolution of radiation of spin weight $s$ in a Kerr black hole spacetime is thus completely described by construction of the source $\mathcal{T}(r)$ appropriate to that spin weight and construction of the homogeneous solutions $R^{H}(r)$ and $R^{\infty}(r)$. As discussed in the Introduction - and, as should be clear from the asymptotic solutions (2.5) and (2.6) - it is very difficult to build these solutions in a numerical integration. The remainder of this paper is devoted to methods for constructing $R^{H}(r)$ and $R^{\infty}(r)$ by finding transformations that relate the Teukolsky solution $R(r)$ to solutions of equations with short-ranged potentials.

\section{RESULTS FOR SCHWARZSCHILD HOLES}

The Teukolsky equation for Schwarzschild black holes is

$$
\Delta^{-s}\left(\Delta^{s+1} R^{\prime}\right)^{\prime}-V_{T S}(r) R=0,
$$

where

$$
V_{T S}(r)=\lambda-4 i s r \omega+\left[2 i s(r-M) \omega-(r \omega)^{2}\right] / f,
$$

and $\lambda=\lambda(a=0)=l(l+1)-s(s+1)$.

We would like to find rules that allow us to obtain $R$ given a solution $X$ of the Regge-Wheeler equation, (1.7). To do so, first define the quantity

$$
\chi \equiv \frac{X}{r \sqrt{\Delta^{s}}} .
$$

If $X$ satisfies the Regge-Wheeler equation, it is straightforward to show that $\chi$ satisfies 


$$
\Delta^{-s}\left(\Delta^{s+1} \chi^{\prime}\right)^{\prime}-U_{\chi S}(r) \chi=0
$$

where

$$
U_{\chi S}(r)=\lambda+\frac{1}{f}\left[s^{2}\left(\frac{3 M^{2}}{r^{2}}-\frac{2 M}{r}\right)-(r \omega)^{2}\right] .
$$

By direct substitution, one can show that $\chi$ can be transformed to $R$, and vice versa, via

$$
\begin{array}{rlrl}
s<0: & \chi & =(r \sqrt{\Delta})^{|s|} \mathcal{D}_{-}^{|s|}\left[\frac{R}{r|s|}\right], \\
& R=\left(\frac{\Delta}{r}\right)^{|s|} \mathcal{D}_{+}^{|s|}\left[\left(\frac{r}{\sqrt{\Delta}}\right)^{|s|} \chi\right], \\
s=0: & \chi & =R, \\
s>0: & \chi & =\left(\frac{r}{\sqrt{\Delta}}\right)^{s} \mathcal{D}_{+}^{s}\left[\left(\frac{\Delta}{r}\right)^{s} R\right], \\
& R=\left(\frac{1}{r}\right)^{s} \mathcal{D}_{-}^{s}\left[(r \sqrt{\Delta})^{s} \chi\right],
\end{array}
$$

where

$$
\mathcal{D}_{ \pm}=d / d r \pm i \omega / f
$$

For $s=-1$ and $s=-2$, Eq. (3.6a) reduces to the Chandrasekhar transformation (see Ref. [11 for $s=-2$, Ref. [6] for $s=-1$ ). Equations (3.6a) - (3.6c) serve as guidelines that will be used to fix the form of the transformation rules for Kerr black holes. Note that the transformations from $R$ to $\chi$ can be written

$$
\chi=\alpha R+\beta \Delta^{s+1} R^{\prime}
$$

by repeatedly using Eq. (3.1) to eliminate derivatives of second order and higher. The resulting functions $\alpha$ and $\beta$ may become rather complicated, particularly for large values of $|s|$, but the general operation is straightforward. (The factor $\Delta^{s+1}$ is inserted for later convenience.)

\section{PERTURBATION EQUATION FOR KERR HOLES}

Guided by Eq. (3.8), let us assume that functions $\alpha$ and $\beta$ can be found that transform the Kerr solution $R$ to solutions $\chi$ of some other equation. By generalizing the relation (3.3) to a form appropriate for the Kerr metric and rewriting all derivatives in terms of $r^{*}$ we will come to an equation with short-ranged potentials governing the behavior of a function $X(r)$. This is the generalized Sasaki-Nakamura (GSN) equation. It will depend explicitly on the (currently unspecified) functions $\alpha$ and $\beta$. These functions will be specified by requiring that the transformation rule satisfy a form which reduces to Eqs. $3.6 \mathrm{a}$ - (3.60) when $a=0$. This guarantees that solutions to the GSN equation are equivalent to solutions of the Regge-Wheeler equation in the Schwarzschild limit.

To begin, differentiate $\chi$ and use Eq. (2.4) to eliminate the second derivative of $R$. The resulting equations for $\chi$ and $\chi^{\prime}$ can be gathered neatly into matrix form:

$$
\left(\begin{array}{c}
\chi \\
\chi^{\prime}
\end{array}\right)=\left(\begin{array}{cc}
\alpha & \beta \Delta^{s+1} \\
\alpha^{\prime}+\beta V_{T} \Delta^{s} & \alpha+\beta^{\prime} \Delta^{s+1}
\end{array}\right)\left(\begin{array}{c}
R \\
R^{\prime}
\end{array}\right) .
$$

A nice feature of Eq. (4.1) is that the inverse solution is rather obvious:

$$
\left(\begin{array}{c}
R \\
R^{\prime}
\end{array}\right)=\frac{1}{\eta}\left(\begin{array}{cc}
\alpha+\beta^{\prime} \Delta^{s+1} & -\beta \Delta^{s+1} \\
-\left(\alpha^{\prime}+\beta V_{T} \Delta^{s}\right) & \alpha
\end{array}\right)\left(\begin{array}{c}
\chi \\
\chi^{\prime}
\end{array}\right)
$$

where

$$
\eta=\alpha\left(\alpha+\beta^{\prime} \Delta^{s+1}\right)-\beta \Delta^{s+1}\left(\alpha^{\prime}+\beta V_{T} \Delta^{s}\right)
$$


is the determinant of the matrix in Eq. (4.1).

Differentiating again and massaging the resultant expression gives us a second-order differential equation for $\chi$ :

$$
\Delta^{-s}\left(\Delta^{s+1} \chi^{\prime}\right)^{\prime}-\Delta_{s} F_{1}(r) \chi^{\prime}-{ }_{s} U_{1}(r) \chi=0 .
$$

The potentials ${ }_{s} F_{1}(r)$ and ${ }_{s} U_{1}(r)$ are given by

$$
\begin{aligned}
& { }_{s} F_{1}(r)=\eta^{\prime} / \eta, \\
& { }_{s} U_{1}(r)=V_{T}+\frac{1}{\beta \Delta^{2}}\left[\left(2 \alpha+\beta^{\prime} \Delta^{s+1}\right)^{\prime}-\frac{\eta^{\prime}}{\eta}\left(\alpha+\beta^{\prime} \Delta^{s+1}\right)\right] .
\end{aligned}
$$

Next, generalize Eq. (3.3) to the Kerr form

$$
\chi \equiv \frac{X}{\sqrt{\left(r^{2}+a^{2}\right) \Delta^{s}}} .
$$

Using this to replace $\chi$ for $X$ in Eq. (4.4) and then replacing derivatives in $r$ with derivatives in $r^{*}$ with the rule

$$
\frac{d}{d r}=\frac{\left(r^{2}+a^{2}\right)}{\Delta} \frac{d}{d r^{*}}
$$

yields the GSN equation:

$$
\frac{d^{2} X}{d r^{* 2}}-{ }_{s} F(r) \frac{d X}{d r^{*}}-{ }_{s} U(r) X=0 .
$$

The potentials are

$$
\begin{aligned}
& { }_{s} F(r)=\frac{\Delta_{s} F_{1}(r)}{r^{2}+a^{2}}, \\
& { }_{s} U(r)=\frac{\Delta_{s} U_{1}(r)}{\left(r^{2}+a^{2}\right)^{2}}+{ }_{s} G(r)^{2}+\frac{\Delta d_{s} G / d r}{r^{2}+a^{2}}-\frac{\Delta_{s} G(r){ }_{s} F_{1}(r)}{r^{2}+a^{2}} .
\end{aligned}
$$

The function ${ }_{s} G(r)$ is

$$
{ }_{s} G(r)=\frac{r \Delta}{\left(r^{2}+a^{2}\right)^{2}}+\frac{s(r-M)}{r^{2}+a^{2}} ;
$$

the functions ${ }_{s} F_{1}(r)$ and ${ }_{s} U_{1}(r)$ are from Eq. (4.5). When $s=-2$, all functions reduce to those given by Sasaki and Nakamura (see Ref. [12]).

All of the quantities which have been derived to this point depend upon the as-yet-undetermined functions $\alpha$ and $\beta$. We fix these functions by requiring that they affect a transformation between $R$ and $\chi$ which, as $a \rightarrow 0$, reduces to Eqs. (3.6a - (3.6d). A useful generalization of these transformations is

$$
\begin{array}{ll}
s<0: & \chi=\left(\sqrt{\left(r^{2}+a^{2}\right) \Delta}\right)^{|s|} g_{0}(r) J_{-}\left[g_{1}(r) J_{-}\left[g_{2}(r) \ldots J_{-}\left[\frac{g_{|s|}(r) R}{\left(\sqrt{r^{2}+a^{2}}\right)^{|s|}}\right]\right]\right], \\
s=0: & \chi=g_{0}(r) R, \\
s>0: & \chi=\left(\sqrt{\frac{r^{2}+a^{2}}{\Delta}}\right)^{s} g_{0}(r) J_{+}\left[g_{1}(r) J_{+}\left[g_{2}(r) \ldots J_{+}\left[g_{s}(r)\left(\frac{\Delta}{\sqrt{r^{2}+a^{2}}}\right)^{s} R\right]\right]\right],
\end{array}
$$

where the operator

$$
J_{ \pm}=d / d r \pm i K(r) / \Delta
$$

generalizes $\mathcal{D}_{ \pm}$to Kerr. The $s=+2$ transformation rule, for example, is

$$
\chi=g_{0}(r) \frac{\left(r^{2}+a^{2}\right)}{\Delta} J_{+}\left[g_{1}(r) J_{+}\left[g_{2}(r) \frac{\Delta^{2}}{r^{2}+a^{2}} R\right]\right] ;
$$


an example for $s=-1$ is given in Sec. V1.

To now specify $\alpha$ and $\beta$, one must pick functions $g_{i}(r)$ and then repeatedly use Eq. (2.4) to eliminate derivatives of second order and higher in Eqs. (4.11a) - (4.11d). The resultant expressions for $\alpha$ and $\beta$ will be, in general, quite complicated; examples are discussed in Secs. $\mathrm{V}$ and $\mathrm{V}$. The functions $g_{i}(r)$ must be chosen so that they become constant in the Schwarzschild limit, and lead to potentials $F(r)$ and $U(r)$ which are short-ranged [i.e., fall off at a rate $O\left(1 / r^{2}\right)$ or faster as $\left.r \rightarrow \infty\right]$. In practice, choosing $g_{i}(r)=1$ or $g_{i}(r)=\left(r^{2}+a^{2}\right) / r^{2}$ appears to lead to well-behaved potentials; some experimentation may be needed to make useful choices.

\section{SCALAR RADIATION}

For scalar radiation, $s=0$, the functions $R, X$, and $\chi$ have the following relationship:

$$
g_{0}(r) R=\chi=\frac{X}{\sqrt{r^{2}+a^{2}}} .
$$

A good choice is $g_{0}(r)=1$. The functions $\alpha$ and $\beta$ [cf. Eq. (3.8)] are then given by

$$
\alpha=1, \quad \beta=0 \text {. }
$$

From this, it follows that

$$
\begin{aligned}
\eta & =1, \\
{ }_{0} F_{1} & =0, \\
{ }_{0} U_{1} & =V_{T}, \\
{ }_{0} G & =\frac{r \Delta}{r^{2}+a^{2}} .
\end{aligned}
$$

The potentials ${ }_{0} F(r)$ and ${ }_{0} U(r)$ are given by substituting Eqs. (5.3a - (5.3d) into Eq. (4.9). For large $r$,

$$
{ }_{0} U(r)=-\omega^{2}+\frac{\lambda+2 a m \omega}{r^{2}}+\frac{2 M(1-\lambda)}{r^{3}}+O\left(1 / r^{4}\right) ;
$$

clearly, ${ }_{0} F(r)=0$ for all $r$. Hence, the potentials are short-ranged. When $a=0,{ }_{0} U(r)$ reduces to $-\omega^{2}+V_{\mathrm{RW}}(r, s=0)$. Thus, it reduces to the Regge-Wheeler equation in the Schwarzschild limit, as it was supposed to.

The asymptotic solutions to the $s=0 \mathrm{GSN}$ equation are simple plane waves:

$$
\begin{aligned}
X^{H}(r) & =e^{-i p r^{*}}, \quad r \rightarrow r_{+}, \\
& =A^{\text {out }} \bar{P}_{0}(r) e^{i \omega r^{*}}+A^{\text {in }} P_{0}(r) e^{-i \omega r^{*}}, \quad r \rightarrow \infty ; \\
X^{\infty}(r) & =C^{\text {out }} e^{i p r^{*}}+C^{\text {in }} e^{-i p r^{*}}, \quad r \rightarrow r_{+}, \\
& =\bar{P}_{0}(r) e^{i \omega r^{*}}, \quad r \rightarrow \infty .
\end{aligned}
$$

The function

$$
P_{0}(r)=1+\frac{\mathcal{A}_{0}}{\omega r}+\frac{\mathcal{B}_{0}}{(\omega r)^{2}}+\frac{\mathcal{C}_{0}}{(\omega r)^{3}}+\ldots
$$

allows us to more accurately describe the behavior of $X^{H, \infty}$ near infinity. This is useful both to improve numerical computations and to derive certain relations between the amplitudes of the Teukolsky solution and the GSN solution. The first three coefficients have the values

$$
\begin{aligned}
\mathcal{A}_{0}= & -\frac{i}{2}(\lambda+2 a m \omega) \\
\mathcal{B}_{0}= & -\frac{1}{8}\left[\lambda^{2}-\lambda(2-4 a m \omega)-4[a m \omega-i M \omega-a m \omega(a m \omega+2 i M \omega)]\right], \\
\mathcal{C}_{0}= & -\frac{i}{6}\left[\mathcal{B}_{0}(\lambda-6+2 a m \omega+8 i M \omega)-4(M \omega)^{2}-2 \mathcal{A}_{0} M \omega(\lambda-6)\right. \\
& \left.-(a \omega)^{2}\left(\lambda-1+m^{2}+2 a m \omega\right)\right] .
\end{aligned}
$$




\section{ELECTROMAGNETIC RADIATION}

For electromagnetic radiation, $s=-1$, the functions $R, X$, and $\chi$ exhibit the following relationships:

$$
\begin{aligned}
\chi & =\sqrt{\frac{\Delta}{r^{2}+a^{2}}} X, \\
\chi & =\alpha R+\beta R^{\prime} \\
& =g_{0}(r) \sqrt{\left(r^{2}+a^{2}\right) \Delta} J_{-}\left[\frac{g_{1}(r) R}{\sqrt{r^{2}+a^{2}}}\right] .
\end{aligned}
$$

From this, we can read off

$$
\begin{aligned}
& \alpha=g_{0} \sqrt{\Delta}\left[g_{1}^{\prime}-\frac{r g_{1}}{r^{2}+a^{2}}-\frac{i g_{1} K}{\Delta}\right], \\
& \beta=g_{0} g_{1} \sqrt{\Delta} .
\end{aligned}
$$

A useful choice for $g_{0}$ and $g_{1}$ is

$$
g_{0}(r)=\frac{\left(r^{2}+a^{2}\right)}{r^{2}}, \quad g_{1}(r)=1
$$

The function $\eta$ that follows from these choices is

$$
\eta=c_{0}+c_{1} / r+c_{2} / r^{2}+c_{3} / r^{3}+c_{4} / r^{4}
$$

where

$$
\begin{aligned}
& c_{0}=-\lambda, \\
& c_{1}=-2 i a m, \\
& c_{2}=a^{2}(1-2 \lambda), \\
& c_{3}=-2 a^{2}(M+i a m), \\
& c_{4}=a^{4}(1-\lambda) .
\end{aligned}
$$

Using this $\eta$ and

$$
{ }_{-1} G(r)=\frac{r \Delta}{\left(r^{2}+a^{2}\right)^{2}}-\frac{(r-M)}{r^{2}+a^{2}},
$$

it is straightforward to construct the functions ${ }_{-1} F_{1}(r),{ }_{-1} U_{1}(r),{ }_{-1} F(r)$, and ${ }_{-1} U(r)$. The results are rather complicated and are not given here. When $r$ is large,

$$
\begin{aligned}
& { }_{-1} F(r)=-\frac{2 i a m}{\lambda r^{2}}+\frac{2 a\left[2 i m M \lambda-a\left(2 m^{2}+2 \lambda^{2}-\lambda\right)\right]}{\lambda^{2} r^{3}}+O\left(1 / r^{3}\right), \\
& { }_{-1} U(r)=-\omega^{2}+\frac{\lambda^{2}+2 a m \omega(\lambda+1)}{\lambda r^{2}}-\frac{2\left[i a^{2}\left(2 m^{2}-\lambda\right)+M\left(\lambda^{3}+2 a m \omega \lambda\right)\right]}{\lambda^{2} r^{3}}+O\left(1 / r^{4}\right) .
\end{aligned}
$$

Both ${ }_{-1} F(r)$ and ${ }_{-1} U(r)$ are short ranged. When $a=0,{ }_{-1} F(r)=0$ and ${ }_{-1} U(r)=-\omega^{2}+V_{R W}(r, s=-1)$.

The solutions $X^{H, \infty}$ are, in the limits $r \rightarrow r_{+}$and $r \rightarrow \infty$, essentially identical to those given in Eqs. (5.5) and (5.6); one need only change the subscript on the $P$ function to -1 . The corresponding coefficients in $P_{-1}(r)$ are

$$
\begin{aligned}
\mathcal{A}_{-1}= & -\frac{i}{2}(\lambda+2 a m \omega) \\
\mathcal{B}_{-1}= & -\frac{1}{8}\left[\lambda^{2}-\lambda(2-4 a m \omega)-4 a \omega\left[m-2 i m M \omega+a \omega\left(2-m^{2}\right)\right]\right], \\
\mathcal{C}_{-1}= & -\frac{i}{6}\left[\mathcal{B}_{-1}(\lambda-6+2 a m \omega+8 i M \omega)+2 \mathcal{A}_{-1}[M \omega(5-\lambda)+i a \omega(2 a \omega+m / \lambda)]\right. \\
& \left.-(a \omega)^{2}\left[\lambda-5+8 i M \omega+2 a m \omega+m^{2}(\lambda+2) / \lambda\right]\right] .
\end{aligned}
$$




\section{CONCLUSION}

In this paper, I have shown how the Sasaki-Nakamura short-ranged equation for gravitational perturbations to a rotating black hole may be generalized to arbitrary spin weight radiation. Of course, there is no particular physical motivation for choosing radiation spin of magnitude greater than 2 ; this approach is taken simply so that one can write down a single rule which encompasses all physically interesting radiation fields, much as the Teukolsky equation itself encompasses all spin weights. Efficient numerical computation of Teukolsky equation solutions now reduces to a simple recipe. First, following the analysis in Sec. IV, develop the potentials needed in the GSN equation, Eq. (4.8). Examples are given for $s=0$ and $s=-1$. Integrate Eq. (4.8) for the GSN solution $X$. Transform to the variable $\chi$ using Eq. (4.6). Then construct the Teukolsky solution $R$ using Eq. (4.1).

One application of these results may be to extend the mode sum regularization scheme described in Ref. [28] to self forces computed in Kerr spacetimes. Calculations that employ scalar or electromagnetic charges and fields are generally simpler than the gravitational self force calculations, which are of great interest for researchers studying gravitational-wave sources. The electromagnetic perturbation equation given in Sec. VI may be of astrophysical interest, particularly when coupled to an appropriate source.

\section{ACKNOWLEDGMENTS}

I thank Lior Burko for encouraging me to find transformation rules and perturbation equations for generic $s$, Eric Poisson for valuable comments, and Manuela Campanelli for pointing me to some useful references. I am also very grateful to Steven Detweiler for valuable comments on the history of black hole perturbation studies which I have used to correct some of the discussion in the Introduction. The package Mathematica was used to aid some of the calculations. This research was supported by NSF Grant AST-9731698 and NASA Grants NAG5-7034 and NAGW-4268.

[1] S. A. Teukolsky, Astrophys. J. 185, 635 (1973).

[2] J. Pullin, Prog. Theor. Phys. Suppl. 136, p. 107 (1999); also gr-qc/9909021.

[3] W. B. Campbell and T. Morgan, Physica 53, 264 (1971).

[4] J. N. Goldberg et al., J. Math. Phys. 8, 2155 (1967).

[5] S. A. Hughes, Phys. Rev. D, in press; also gr-qc/9910091.

[6] S. W. Leonard and E. Poisson, Phys. Rev. D 56, 4789 (1997).

[7] S. A. Teukolsky and W. H. Press, Ap. J. 193, 443 (1974).

[8] W. H. Press and S. A. Teukolsky, Ap. J. 185, 649 (1973).

[9] S. Chandrasekhar, Proc. R. Soc. London A343, 289 (1975).

[10] E. W. Leaver, Proc. Roy. Soc. London A402, 285 (1985).

[11] E. Poisson, Phys. Rev. D 47, 1497 (1993).

[12] M. Sasaki and T. Nakamura, Phys. Lett. 89A, 68 (1982); M. Sasaki and T. Nakamura, Prog. Theor. Phys. 67, 1788 (1982).

[13] S. Chandrasekhar and S. L. Detweiler, Proc. Roy. Soc. London A350, 165 (1976).

[14] S. L. Detweiler, Proc. Roy. Soc. London A352, 381 (1977).

[15] S. Chandrasekhar, in General Relativity - An Einstein Centenary Survey, edited by S. W. Hawking and W. Israel (Cambridge, England, 1979), chap. 7.

[16] Much of the work by Chandrasekhar and collaborators is surveyed in S. Chandrasekhar, The Mathematical Theory of Black Holes (Oxford University Press, New York, 1983), chaps. 8 - 9.

[17] S. Detweiler, private communication.

[18] M. Campanelli and C. O. Lousto, Phys. Rev. D 56, 6363 (1997).

[19] Y. Kojima and T. Nakamura, Phys. Lett. 96A, 335 (1983); Y. Kojima and T. Kakamura, Prog. Theor. Phys. 71, 79 (1984).

[20] M. Shibata, Phys. Rev. D 50, 6297 (1994).

[21] Y. Mino et al., Prog. Theor. Phys. Supplement No. 128 (1997), chap. 1.

[22] D. Kennefick, Phys. Rev. D 58, 4012 (1998); also gr-qc/9805102.

[23] Y. Mino, M. Sasaki, and T. Tanaka, Phys. Rev. D 55, 3457 (1997).

[24] T. C. Quinn and R. M. Wald, Phys. Rev. D 56, 3381 (1997).

[25] W. G. Anderson, E. E. Flanagan, and A. C. Ottewill, in preparation. 
[26] A. G. Wiseman, Phys. Rev. D, in press; also gr-qc/0001025.

[27] A. Ori, Phys. Lett. A 202, 347 (1995); A. Ori, Phys. Rev. D 55, 3444 (1997).

[28] L. Barack and A. Ori, Phys. Rev. D, in press; also gr-qc/9912010.

[29] L. M. Burko, Class. Quantum Grav. 17, 227 (2000).

[30] L. M. Burko, in preparation; see also L. M. Burko, gr-qc/9911089.

[31] G. Arfken, Mathematical Methods for Physicists (Academic Press, Orlando, 1985), chap. 16. 